\title{
The Pompe Registry: tracking Pompe disease symptoms in a broad
} patient population

\author{
B Byrne1, PS Kishnani², L Case², L Merlini³, W Müeller-Felber, A Van \\ der Ploeg ${ }^{5}$ D Marsden ${ }^{6}$ and S Prasad ${ }^{* 6}$
}

\begin{abstract}
Address: ${ }^{1}$ Congenital Heart Center University of Florida, College of Medicine, Gainesville, FL, USA, ${ }^{2}$ Duke University Medical Center, Durham, NC, USA, ${ }^{3}$ Department of Medical Genetics, University of Ferrara, Ferrara, Italy, ${ }^{4}$ Department of Neurology, University of Munich, Munich, Germany, ${ }^{5}$ Erasmus Medical Center, Sophia,, Rotterdam, Netherlands and ${ }^{6}$ Genzyme Corporation, Cambridge, MA, USA

* Corresponding author
\end{abstract}

from I5 ${ }^{\text {th }}$ Paediatric Rheumatology European Society (PreS) Congress

London, UK. 14-17 September 2008

Published: 15 September 2008

Pediatric Rheumatology 2008, 6(SuppI I):PI59 doi:10.II86/1546-0096-6-SI-PI59

This abstract is available from: http://www.ped-rheum.com/content/6/SI/PI59

(C) 2008 Byrne et al; licensee BioMed Central Ltd.

\section{Introduction}

Pompe disease is a rare, progressive, often fatal metabolic myopathy, which manifests as a clinical spectrum that varies with respect to age at onset, rate of disease progression, and extent of organ involvement. The underlying pathology is deficiency of acid alpha-glucosidase (GAA). To gain a better understanding of Pompe disease, a global, voluntary, observational Registry was developed to collect anonymous, longitudinal data.

\section{Preliminary data overview}

As of March 2008, 494 patients from 23 countries were enrolled; the majority (72\%) Caucasian. Europe and North America enroll $87 \%$ of patients. Median age of infants at symptom onset was 2.0 months $(n=94)$ and at diagnosis was 4.0 months $(n=93)$. Median age of adults at symptom onset was 27.7 years $(n=293)$ and at diagnosis was 35.3 years $(n=289)$. Symptoms most frequently reported by patients $\geq 18$ years old $(n=321)$ include: muscle weakness [lower extremities (80\%), upper extremities $(69 \%)$, trunk (53\%)]; shortness of breath after exercise $(61 \%)$ and at rest (31\%); dependence on respiratory support (38\%); sleep disturbance/apnea (35\%); orthopnea (32\%); and scapular winging (31\%). Approximately half of patients genotyped expressed the IVS1-13T>>G mutation.

\section{Summary}

These results show significant delay from symptom onset to diagnosis in adult patients, highlighting the need for greater disease awareness. Registry data on prevalence and age at onset of symptoms may allow earlier patient identification, enabling intervention before irreversible muscle damage occurs. Analysis of registry data over time may increase understanding of the evolution of, and interaction between, impairments and function under varying conditions and interventions, allowing improved clinical management. 